${ }^{1}$ Carrera de Nutrición y Dietética. Facultad de Farmacia, Universidad de Valparaíso. Chile.

${ }^{2}$ Escuela de Nutrición. Facultad de Farmacia, Universidad de Valparaíso. Chile.

${ }^{3}$ Departamento de Psiquiatría, Hospital del Salvador, Valparaíso. Chile.

aNutricionista.

Sin fuente de financiamiento.

Recibido el 15 de junio de 2016, aceptado el 31 de agosto de 2016.

Correspondencia a: Nta. Claudia Vega Soto Escuela de Nutrición, Facultad de Farmacia, Universidad de Valparaíso. Av Gran Bretaña 1093, Correo 7 Valparaíso, Chile. Tel (5632) 2508440 Fax (5632) 2508111 claudia.vega@uv.cl

\section{Ingesta de hidratos de carbono y ácidos grasos de la dieta en sujetos con esquizofrenia y trastorno bipolar, y su asociación con parámetros antropométricos}

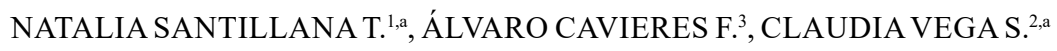

Background: People with psychiatric disorders have higher rates of obesity, diabetes mellitus and dyslipidemia. These comorbidities are associated with the underlying psychopathology and drug therapy. Aim: To determine the quality and quantity of carbohydrates and fatty acids in the diet and their association with anthropometric parameters in subjects with schizophrenia and bipolar disorders. Patients and Methods: We studied 30 patients with schizophrenia and bipolar disorders in treatment with atypical antipsychotics or mood stabilizers. Three 24-hour recall dietary surveys were carried out. Glycemic index, intake of carbohydrates and fatty acids (g/day) were calculated, and the ratio of saturated, monounsaturated and polyunsaturated fatty acids was determined. Body mass index, waist circumference (WC) and body fat percentage were evaluated. Results: The average intakes of carbohydrates and fatty acids were $295 \pm 111$ and $73 \pm 38 \mathrm{~g} /$ day respectively. The mean glycemic index was $59 \% \pm 5.4$, while the ratio of saturated, monounsaturated and polyunsaturated fatty acids was 2: 1.4: 0.6. No association between dietary and anthropometric variables was found. Patients using second-generation antipsychotics had a significantly higher waist circumference than those using mood stabilizing drugs. Conclusions: We found no association between the amount and quality of carbohydrate or fatty acid dietary intake and anthropometric parameters.

(Rev Med Chile 2016; 144: 1164-1170)

Key words: Anthropometry; Bipolar Disorder; Dietary Carbohydrates; Fatty Acids; Schizophrenia.

L as personas con esquizofrenia y trastorno bipolar tienen menor esperanza de vida en comparación con la población general ${ }^{1}$. Las causas de mortalidad se deben fundamentalmente al suicidio y a sucesos naturales, entre los que destacan eventos cardiovasculares ${ }^{2}$. Los pacientes con trastorno psiquiátrico presentan altas tasas de diabetes mellitus, hipertensión arterial, obesidad y dislipidemia ${ }^{3,4}$.

Estudios han observado que el índice de masa corporal (IMC) promedio en pacientes con trastorno bipolar es de $27,7 \pm 6,2 \mathrm{~kg} / \mathrm{m}^{2}$, mientras que en esquizofrenia sería de $28,9 \pm 3,2 \mathrm{~kg} / \mathrm{m}^{2}$ y una circunferencia de cintura (CC) promedio de 100,8 
(SD 9,2) $\mathrm{cm}^{6}$. Dentro de los factores etiológicos incide la predisposición genética y la psicopatología subyacente ${ }^{7}$. Los efectos adversos del tratamiento farmacológico, a través de antipsicóticos atípicos, también podrían contribuir al desarrollo de enfermedades cardiovasculares (ECV) en estos pacientes. El uso de estos medicamentos se ha asociado con un drástico aumento de peso, diabetes mellitus y un perfil lipídico aterogénico ${ }^{8}$.

Sin embargo, y acorde a la situación nutricional actual, se observan en estos sujetos conductas modificables, como el tabaquismo, sedentarismo, e inadecuada alimentación ${ }^{7}$. En pacientes con trastornos psiquiátricos, se ha visto que quienes tienden a comer una gran cantidad de ácidos grasos saturados (AGS), en general, tienden a consumir mucha azúcar?.

Según el patrón alimentario descrito anteriormente, estos sujetos presentan un mayor riesgo de desarrollar ECV debido al exceso de ingesta de energía, hidratos de carbono ( $\mathrm{CHO}$ ) simples y AGS, lo cual, además, constituye un factor de riesgo para desarrollar enfermedades crónicas ${ }^{10}$. Los $\mathrm{CHO}$ son unos de los macronutrientes que aportan la mayor proporción de energía de la dieta, y por lo tanto, pueden contribuir al aumento de peso cuando se consumen en exceso en relación a las necesidades energéticas ${ }^{11}$. Uno de los indicadores de calidad de los $\mathrm{CHO}$ de la dieta es el índice glicémico (IG). El IG, consiste en comparar el área bajo la curva de la respuesta glicémica en vivo de un alimento, respecto a un alimento patrón (glucosa o pan blanco) y se expresa como porcentaje de éste (se utiliza una carga de $50 \mathrm{~g}$ de $\mathrm{CHO}$ disponibles) ${ }^{12}$. El IG obedece a la necesidad de distinguir el efecto fisiológico de los $\mathrm{CHO}$ independientemente de la complejidad de su estructura (simple o compleja ${ }^{13}$. Estudios han sugerido que dietas con bajo IG pueden ayudar a prevenir el aumento de peso corporal, estimular la pérdida de peso y reducir la grasa corporal ${ }^{14}$.

Los ácidos grasos (AG) son uno de los factores de mayor incidencia sobre la densidad energética de la dieta. El consumo excesivo de grasas se ha relacionado con riesgo de obesidad y de $\mathrm{ECV}^{15}$. Un indicador de calidad de la grasa es la relación de AGS, ácidos grasos monoinsaturados (AGMI) y ácidos grasos poliinsaturados (AGPI). Una inadecuada distribución de AG de la dieta o una mayor ingesta de AGS en desmedro de AGMI y AGPI es un factor de riesgo para ECV. La Food and Agriculture Organization (FAO) recomienda para la población general una distribución 1:1:1 de estos nutrientes ${ }^{16}$.

Existe evidencia suficiente que describe el patrón alimentario de los sujetos con trastornos psiquiátricos, sin embargo, no hay estudios claros que permitan asociar la ingesta dietética con los parámetros antropométricos de éstos. La hipótesis planteada en este estudio es que existe asociación entre la cantidad y calidad de los CHO y AG de la dieta con IMC, CC y porcentaje de grasa corporal en sujetos con esquizofrenia y trastorno bipolar.

En base a estos antecedentes, el objetivo de este estudio fue describir la ingesta de CHO y AG de la dieta y asociarlo con parámetros antropométricos en sujetos con esquizofrenia y trastorno bipolar. Además se agruparon los pacientes según tipo de tratamiento para compararlos en relación a la antropometría.

\section{Pacientes y Método}

Se evaluaron 30 sujetos mayores de 18 años pertenecientes a la Corporación Dr. Carlos Bresky Viña del Mar Chile, diagnosticados de esquizofrenia o trastorno bipolar estables en su condición clínica, en tratamiento mayor a 6 meses con antipsicóticos o estabilizadores de ánimo $y$ con IMC $\geq a 18,5 \mathrm{~kg} / \mathrm{m}^{2}$. Se excluyeron de este estudio aquellos sujetos que presentaron comorbilidades médicas severas, mujeres embarazadas $y$ analfabetos. Todos los pacientes firmaron un consentimiento informado antes de ingresar al estudio, donde se les explicó el propósito de la investigación, la voluntariedad de la participación y la confidencialidad. Este trabajo fue aprobado por el Comité de Ética de la Facultad de Farmacia de la Universidad de Valparaíso.

\section{Determinaciones}

\section{Antropometría}

Se registró el peso corporal con una balanza digital Tanita ${ }^{\oplus}$ (Tanita HD ${ }^{\oplus} 351$ Digital Weight Scale, Tokyo, Japón. Precisión de 0,1 kg). La talla se determinó con un estadiómetro Seca ${ }^{\varpi}$ Seca $^{\varpi}$ 213, Hamburgo, Alemania. Precisión de $1 \mathrm{~mm}$ ). Las mediciones fueron realizadas con los sujetos descalzos, con el peso distribuido uniformemente y con ropa ligera. Con los datos obtenidos se 
calculó el IMC $\left(\mathrm{IMC}=\text { peso }[\mathrm{kg}] / \text { talla }\left[\mathrm{m}^{2}\right]\right)^{17}$. Se evaluó CC utilizando una cinta métrica marca Seca ${ }^{\circledast}$ Seca $^{\circledR}$ 201, Hamburgo, Alemania. Sensibilidad $1 \mathrm{~mm}$ ), justo inmediatamente sobre la cresta ilíaca. Los valores de la CC se clasificaron de acuerdo a la International Diabetes Federation (IDF), representando valores $\geq 90 \mathrm{o} \geq 80 \mathrm{~cm}$ en hombres y mujeres, respectivamente, un alto riesgo de $\mathrm{ECV}^{18}$. Además, se estimó el porcentaje de masa grasa empleando bioimpedanciómetro tetrapolar Bodystat ${ }^{\circledast}$ (Bodystat ${ }^{\circledR}$ 1500,Isla de Man, Gran Bretaña. Precisión Impedancia 2-3).

\section{Ingesta dietética}

Se realizaron tres encuestas de recordatorio de 24 h de dos días de la semana y uno de fin de semana por un profesional nutricionista, quien realizó el correcto registro de la información, considerando horarios, tiempos de comida, preparaciones y cantidad de alimentos consumidos. Las encuestas fueron analizadas en el programa computacional FoodProcessor II ${ }^{\circledast}$ (FoodProcessor SQL 10.7. ESHA Research, Salem, OR, USA), que utiliza una base de datos de composición química de alimentos norteamericanos, a la cual se le agregaron alimentos chilenos, para evaluar y cuantificar la cantidad y calidad de los CHO y AG de la dieta. Además, para estimar la suficiencia de la alimentación se consignaron los siguientes indicadores dietéticos:

- Índice glicémico: Para determinar la calidad de los CHO (ecuación 1) ${ }^{19}$.

Ecuación 1: cálculo del índice glicémico.

$\mathrm{IG}=($ gramos de $\mathrm{CHO}$ de cada $\mathrm{tc} / \mathrm{CHO} \mathrm{d}$ totales de la dieta) $x$ (IG de cada tc)

Donde: $\mathrm{IG}=$ índice glicémico; $\mathrm{CHO} \mathrm{d}=$ hidratos de carbono disponibles; $\mathrm{tc}=$ tiempo de comida.

De acuerdo a Brand Miller y cols, se clasificaron los tiempos de comidas según las siguientes categorías: bajo IG $\leq 55$, IG moderado 56-69, y alto $I G \geq 70^{20}$.

- Relación de ácidos grasos AGS: AGMI:AGPI: Para evaluar la calidad de los AG de la dieta.

- Relación de $\mathrm{CHO} / \mathrm{kg}$ peso ideal y $\mathrm{AG} / \mathrm{kg}$ peso ideal: Para estimar la ingesta de CHO y AG de la dieta en relación al peso ideal de cada sujeto.

\section{Análisis estadístico}

Se determinó la distribución normal de las variables con el test de Shapiro-Wilk. Los resultados se expresaron como promedio \pm desviación estándar. Se aplicó la prueba de correlación de Pearson para determinar la asociación entre las variables dietéticas y antropométricas. Se clasificaron los sujetos según el tipo de tratamiento farmacológico, para luego determinar diferencias en las variables antropométricas utilizando ANOVA de una vía. El análisis estadístico fue realizado con el programa computacional SPSS 17.0 (SPSS Inc., Chicago IL, USA). Se consideró significativo un $\mathrm{p}<0,05$ para todos los análisis.

\section{Resultados}

Las características generales y antropométricas de los sujetos estudiados aparecen en las Tablas 1 y 2 , respectivamente. La edad mínima de los sujetos fue de 19 años y la máxima de 69 años. El $85 \%$ de los sujetos tenía el diagnostico psiquiátrico de esquizofrenia o trastorno bipolar hacía más de un año. Del total de pacientes en tratamiento con antipsicóticos atípicos, 69\% estaba con clozapina, $7 \%$ con olanzapina, $10 \%$ con risperidona y $7 \%$ con aripiprazol. Todos los pacientes con estabilizador de ánimo estaban en tratamiento con lamotrigina.

En relación a la antropometría, $73 \%$ de los sujetos presentaba mal nutrición por exceso; $28 \%$ tenía sobrepeso y $45 \%$ obesidad. Con respecto a la circunferencia de cintura, $79 \%$ de las personas presentaba alto riesgo cardiovascular (RCV). Por otra parte, $85 \%$ de las mujeres y $75 \%$ de los hombres, presentaban exceso de grasa corporal.

\section{Tabla 1. Características demográficas de los sujetos estudiados}

\begin{tabular}{|lc|}
\hline Parámetro & Valor \\
\hline Sujetos estudiados $(\mathrm{n})$ & 30 \\
\hline Edad (años; Promedio \pm DE) & $39 \pm 13$ \\
Género ( $\mathrm{n})$ & \\
$\quad$ Femenino & 14 \\
\hline Diagnóstico psiquiátrico $(\mathrm{n})$ & \\
$\quad$ Esquizofrenia & 22 \\
$\quad$ Trastorno bipolar & 8 \\
\hline Tratamiento farmacológico (n) & \\
$\quad$ Antipsicótico atípico & 21 \\
$\quad$ Antipsicótico atípico + ansiolítico & 5 \\
Estabilizador de ánimo & 4 \\
\hline
\end{tabular}

n: número de personas. 
Tabla 2. Características antropométricas de los sujetos estudiados

\begin{tabular}{|c|c|}
\hline Variables & Valor \\
\hline Peso $^{a}(k g)$ & $79,7 \pm 19,3$ \\
\hline Talla $(m)$ & $1,6 \pm 0,1$ \\
\hline $\mathrm{IMC}^{\mathrm{a}}\left(\mathrm{kg} / \mathrm{m}^{2}\right)$ & $29,5 \pm 7,0$ \\
\hline \multicolumn{2}{|l|}{ Estado nutricional } \\
\hline $\begin{array}{l}\text { Normal (n) } \\
\text { Sobrepeso (n) } \\
\text { Obesidad clase I (n) } \\
\text { Obesidad clase II (n) } \\
\text { Obesidad clase III (n) }\end{array}$ & $\begin{array}{l}8 \\
9 \\
8 \\
3 \\
2\end{array}$ \\
\hline $\begin{array}{l}\text { Circunferencia de cintura }{ }^{\mathrm{a}}(\mathrm{cm}) \\
\text { Hombres }^{\mathrm{a}} \\
\text { Mujeres }^{\mathrm{a}}\end{array}$ & $\begin{aligned} 99,8 & \pm 17,9 \\
100,2 & \pm 12,4 \\
99,5 & \pm 23,1\end{aligned}$ \\
\hline $\begin{array}{l}\text { Masa grasa }{ }^{a}(\%) \\
\text { Hombres }^{a} \\
\text { Mujeres }^{a}\end{array}$ & $\begin{array}{l}33,6 \pm 11,4 \\
28,4 \pm 5,3 \\
31,6 \pm 13,7\end{array}$ \\
\hline
\end{tabular}

${ }^{a}$ Datos expresados como promedios \pm desviación estándar (DE). n: número de personas. IMC: índice de masa corporal.
Tabla 3. Cantidad y calidad de los CHO y AG de la dieta de los sujetos estudiados

\begin{tabular}{|lc|}
\hline Variables & Promedio \pm DE \\
CHO (g) & $295 \pm 111$ \\
CHO (\%) & $51 \pm 8$ \\
CHO (g) /kg peso ideal & $5 \pm 2$ \\
IG (\%) & $59 \pm 5,4$ \\
AG (g) & $73 \pm 38$ \\
AG (\%) & $28 \pm 9$ \\
AG (g) /kg peso ideal & $1,3 \pm 1$ \\
AGS (g) & $16 \pm 8$ \\
AGMI (g) & $18 \pm 13$ \\
AGPI (g) & $14 \pm 10$ \\
\hline
\end{tabular}

a:Datos expresados como promedios $\pm \mathrm{DE}$. $\mathrm{CHO}$ : hidratos de carbono; IG: índice glicémico; AG: ácidos grasos; AGS: ácidos grasos saturados; AGMI: ácidos grasos monoinsaturados; AGPI: ácidos grasos poliinsaturados.

Tabla 4. Antropometría según tipo de tratamiento farmacológico de los sujetos estudiados

\begin{tabular}{|ccccc|}
\hline Antropometría & T1 (n= 21) & T2 (n = 5) & T3 (n = 4) & p* $^{*}$ \\
IMC $\left(\mathrm{kg} / \mathrm{m}^{2}\right)$ & $30,3 \pm 7$ & $26,9 \pm 6$ & $21 \pm 1$ & $\mathrm{NS}$ \\
CC (cm) & $105,2 \pm 14^{\mathrm{a}}$ & $94,2 \pm 22$ & $78,6 \pm 23^{\mathrm{b}}$ & 0,021 \\
\% MG & $34 \pm 12$ & $34,2 \pm 14$ & $30,4 \pm 7$ & $\mathrm{NS}$ \\
\hline
\end{tabular}

Datos expresados como promedios $\pm \mathrm{DE}$. *Significancia estadística $p<0,05$ según método de ANOVA de un factor. $a$, b: Letra distinta en superíndice indica diferencias significativas entre los tratamientos. IMC: índice de masa corporal; CC: circunferencia de cintura; \% MG: porcentaje masa grasa.T1: Antipsicótico atípico; T2: Antipsicótico atípico + ansiolítico; T3: Estabilizador de ánimo.

Los análisis de las encuestas alimentarias se muestran en la Tabla 3. El 60\% de los sujetos tenía una ingesta de energía proveniente de los $\mathrm{CHO}$ entre 50 y $60 \%$ de las calorías totales. La ingesta mínima de $\mathrm{CHO}$ fue de $121 \mathrm{~g}$ y la máxima de 656 g. Respecto a la ingesta en relación al peso ideal, la mínima fue de $2 \mathrm{~g}$ de $\mathrm{CHO} / \mathrm{kg}$ peso ideal y la máxima de $11 \mathrm{~g}$ de $\mathrm{CHO} / \mathrm{kg}$ peso ideal. En relación a la calidad de los $\mathrm{CHO}, 89,6 \%$ de los sujetos consumía una dieta de moderado IG (55-70\%).

El porcentaje de calorías provenientes de los AG fue de $29 \pm 9 \%$. El $23 \%$ de los pacientes consumía entre 0,8 y $1 \mathrm{~g}$ de ácidos grasos $/ \mathrm{kg}$ peso ideal. Siendo la mínima 0,4 AG/kg de peso ideal y máxima 3,7 g de AG/kg de peso ideal. La relación de los AGS:AGMI:AGPI de la dieta fue de 2:1,4:0,6.

No se observó correlación entre las variables dietéticas y antropométricas. Por otro lado, se encontró que la CC de los pacientes con antipsicóticos atípicos se diferencia significativamente de la CC de los pacientes con estabilizadores de ánimo $(\mathrm{p}<0,05)($ Tabla 4$)$.

\section{Discusión}

La gran mayoría de la muestra estudiada presenta malnutrición por exceso, lo cual es similar 
a lo reportado por Inamura et al., de los 35 pacientes estudiados, 18 presentaban un IMC igual o mayor a $25 \mathrm{~kg} / \mathrm{mt}^{2}{ }^{6}$. Asimismo, Archie y cols. hallaron que $36 \%$ de su muestra tenía obesidad $\left(\mathrm{IMC} \geq 30 \mathrm{~kg} / \mathrm{mt}^{2}\right)^{21}$.

En relación a la obesidad central, se observó que $79 \%$ de los pacientes presentaba alto RCV según CC. Esto es coherente con el estudio realizado por Nunes et $\mathrm{al}^{22}$, donde $70 \%$ de los participantes presentaba alto RCV. Si bien, Nunes et al., midieron la CC en la zona más estrecha del tronco, la determinación de la CC justo por sobre la cresta ilíaca representa un valor de utilidad clínica. La literatura sugiere que este indicador predice en igual forma el RCV que con la CC central. Con este último punto de medición, el valor promedio de CC para la población chilena es de $95,1 \mathrm{~cm}$ para los hombres y de $95,9 \mathrm{~cm}$ para las mujeres. Con mediciones de CC realizadas por encima de la cadera, y tomando en cuenta puntos de corte propuestos por la IDF, la obesidad abdominal en Chile está presente en $45,1 \%$ de los hombres y en $71,5 \%$ de las mujeres ${ }^{23}$.

Por otra parte, el valor promedio del porcentaje de masa grasa de este estudio $(33,8 \pm 11,7 \%)$ es menor a lo observado por Hassapidou et al, quienes en el total de los pacientes observaron un porcentaje de grasa corporal promedio de 38,3 \pm $8,03 \%{ }^{24}$.

En relación a la ingesta dietética, el 59\% de los sujetos tenía una ingesta de energía proveniente de los $\mathrm{CHO}$ acorde a las recomendaciones de consumo para la población general. El consumo promedio de CHO observado en este estudio fue de $295 \pm 111 \mathrm{~g}$, valor mayor a lo que obtuvieron Henderson et al., de 236,7 $\pm 104,6$ g y que sorprendentemente era menor respecto a su grupo control ${ }^{25}$. Con respecto al IG, cerca de $90 \%$ de los pacientes consumía una dieta de moderado IG. El valor promedio encontrado (59\%) coincide con lo determinado por Blye et $\mathrm{al}^{26}$.

El consumo promedio de calorías provenientes de los AG fue de 28,9 $\pm 9 \%$, porcentaje menor a lo observado por Henderson et $\mathrm{al}^{25,27}$. Sin embargo, mayor a lo reportado por Inamura et $\mathrm{al}^{6}$, en sujetos obesos y no obesos con esquizofrenia. En esta investigación se encontró que los AGS se consumían en mayor proporción, ninguno de los pacientes estudiados cumplía la recomendación establecida por la $\mathrm{FAO}^{16}$. Cabe destacar que, en la literatura, se hace mayor énfasis a la relación
AGMI y AGPI, o a la relación $n-6 / n-3$, y se ha visto que en sujetos con trastorno bipolar hay una menor ingesta de AGPI con un elevado consumo de $\mathrm{AGS}^{28}$.

En este estudio no se encontró asociación alguna entre la dieta y los parámetros antropométricos. No existen estudios claros que correlacionen estos parámetros. Blye et $\mathrm{al}^{26}$, en un modelo de regresión, examinaron la relación entre el síndrome metabólico y parámetros dietéticos, en pacientes con esquizofrenia y trastorno bipolar, no obstante, ninguno de los parámetros dietéticos y de estilos de vida se asociaron con el síndrome metabólico.

Por otra parte, al agrupar los pacientes por tipo de tratamiento, las personas que estaban con antipsicóticos atípicos presentaron mayor IMC, CC y porcentaje de grasa corporal, en comparación a las otras terapias. Se ha señalado incremento del depósito de grasa subcutánea y abdominal, así como aumento de peso con el uso de estos medicamentos ${ }^{29}$. Se encontraron diferencias significativas en la $\mathrm{CC}$ en pacientes que estaban con antipsicóticos atípicos y estabilizador de ánimo. La CC era mayor en los sujetos con antipsicóticos de segunda generación. Existe evidencia actual que relaciona los niveles de obesidad con el uso de antipsicóticos atípicos, que generan un mayor aumento de peso que otros tratamientos de monoterapia (antipsicóticos típicos y estabilizadores de ánimo). Se plantea que fármacos como clozapina y olanzapina tendrían un mayor efecto sobre el peso. Se ha postulado que inducen algunos cambios en la conducta alimentaria como aumento del apetito y disminución de la saciedad ${ }^{30}$. El alza de peso se puede explicar por el efecto antagonista a receptores de serotonina 5HT2 $\mathrm{C}$ y receptores $\mathrm{H} 1$ de histamina. La acción de estos fármacos como antagonistas de los receptores 5HT2C da como resultado el aumento de expresión del neuropéptido Y (NPY) y de la hormona concentradora de melanina del receptor 1 (MCHR1); la acción antagonista a receptores $\mathrm{H} 1$ de histamina, se traduce en la reducción de la lipólisis en el tejido adiposo blanco al disminuir la activación de AMPK, proteína que en su forma activa, juega un rol importante en el metabolismo energético. La activación de AMPK se asocia con una disminución de la formación de lípidos, porque AMPK fosforila acetil-CoAcarboxilasa (ACC) inhibiendo la generación de malonil-CoA. Malonil-CoA es un sustrato para la enzima ácido graso sintasa ${ }^{31}$. Este aumento de 
apetito y disminución de la lipólisis se traduce en un evidente incremento en el peso ${ }^{32}$. Esto da como resultado un aumento en la circunferencia de cintura, al depositarse grasa a nivel abdominal y visceral, constituyendo un $\mathrm{RCV}^{33}$.

En conclusión, este estudio muestra que no existe asociación entre la cantidad y la calidad de los CHO y AG de la dieta con los parámetros antropométricos en sujetos con trastorno bipolar y esquizofrenia. Sin embargo, los sujetos que están en tratamiento con antipsicóticos de segunda generación presentan una CC significativamente mayor que los sujetos que están en tratamiento con estabilizadores de ánimo. Si bien, no se encontró asociación con la ingesta dietética, se hace necesaria la promoción de estilos de vida saludables en estos pacientes, para lograr pérdida de peso, resultando en menor CC y así menor RCV.

Agradecimientos: Los autores expresan su agradecimiento a Informantes Paulina Ibaceta y Ana María Neira por su colaboración en la revisión del manuscrito. Igualmente se agradece la colaboración del personal técnico-administrativo y profesional de la Corporación Dr. Carlos Bresky Viña del Mar Chile, por su participación en la identificación de potenciales candidatos para el estudio.

\section{Referencias}

1. Chang CK, Hayes RD, Broadbent M, Fernandes AC, Lee W, Hotopf M, et al. All-cause mortality among people with serious mental illness (SMI), substance use disorders, and depressive disorders in southeast London: a cohort study. BMC Psychiatry 2010; 10 (1): 77.

2. Morden NE, Mistler LA, Weeks WB, Bartels SJ. Health Care for Patients with Serious Mental Ilness: Family Medicine's Role. J Am Board FamMed 2009; 22 (2): 187-95.

3. Bresee LC, Majumdar SR, Patten SB, Johnson JA. Prevalence of cardiovascular risk factors and disease in people with schizophrenia: a population-based study. Schizophr Res 2010; 117 (1): 75-82.

4. Weiner M, Warren L, Fiedorowicz JG. Cardiovascular morbidity and mortality in bipolar disorder. Ann Clin Psychiatry 2011; 23 (1): 40-7.

5. Wang PW, Sachs GS, Zárate CA, Marangell LB, Calabrese JR, Goldberg JF, et al. Overweight and obesity in bipolar disorders. J Psychiatr Res 2006; 40 (8): 762-4.

6. Inamura $\mathrm{Y}$, Izumi T, Sakurai K, Katsui T, Murayama N.
Dietary factors associated with obesity in patients with shizophrenia living at home. Food Nutr Sci 2012; 3 (10): 1387-96.

7. Osborn DP, Levy G, Nazareth I, Petersen I, Islam A, King MB. Relative risk of cardiovascular and cancer mortality in people with severe mental illness from the United Kingdom's General Practice Research Database. Arch Gen Psychiatry 2007; 64 (2): 242-9.

8. American Diabetes Association, American Psychiatric Association, American Association of Clinical Endocrinologists, North American Association for the Study of Obesity. Consensus Development Conference on Antipsychotic Drugs and Obesity and Diabetes. Diabetes Care 2004; 27 (2): 596-601.

9. Amani R. Is dietary pattern of schizophrenia patients different from healthy subjects? BMC Psychiatry 2007; 7 (1): 15

10. Burges A, Glassauer P, editores. Guía de nutrición de la familia. 7ma ed. Roma: FAO; 2006.

11. Mann J, Cummings JH, Englyst HN, Key T, Liu S, Riccardi G, et al. FAO/WHO scientific update on carbohydrates in human nutrition: conclusions. Eur J Clin Nutr 2007; 61 (1): 132-7.

12. Jenkins DJ, Wolever TM, Taylor RH, Barker H, Fielden $\mathrm{H}$, Baldwin JM, et al. Glycemic index of foods: a physiological basis for carbohydrate exchange. Am J Clin Nutr 1981; 34 (3): 362-6.

13. Jenkins DJ, Jenkins AL, Wolever TM, Collier GR, Rao AV, Thompson LU, et al. Starchy foods and fiber: reduced rate of digestion and improved carbohydrates metabolism. Scand J Gastroenterol Suppl 1987; 129: 132-41.

14. Feliciano P, Graçasde Almeida C, Gonçalves R de C. Glycemic index role on visceral obesity, subclinical inflamation and associated chronic disseases. Nutr Hosp 2014; 30 (2): 237-43.

15. Torrejón C, Uauy R. Calidad de grasa, arterioesclerosis y enfermedad coronoria: efecto de los ácidos grasos saturados y ácidos grasos trans. Rev Med Chile 2011; 139: 924-31.

16. Food and Agriculture Organization of the United Nations. Fats and fatty acids in human nutrition. Report of an expert consultation. FAO Food Nutr Pap 2010; 91: $1-166$.

17. WHO. Obesity: preventing and managing the global epidemic. Report of a WHO Consultation. WHO Technical Report Series 894. Geneva: World Health Organization, 2000.

18. International Diabetes Federation. The IDF consensus worldwide definition of the metabolic syndrome. IDF Communications 2006; 1-16. 
19. Wolever TM, Brand-Miller JC, Abernethy J, Astrup A, Atkinson F, Axelsen M, et al. Measuring the glycemic index of foods: inter laboratory study. Am J Clin Nutr 2008; 87 (1): 247-57.

20. Brand Miller J, Wolever T M, Foster Powell, et al The New Glucose Revolution Nueva York, NY: Moerlowe \& Company; 2003.

21. Archie SM, Goldber JO, Akhtar-Danesh N, Landeen J, McColl L, McNiven J. Psychotic Disorders, Eating Habits, and Physical Activity: Who Is Ready for Lifestyle Changes? Psychiatr Serv 2007; 58 (2): 233-9.

22. Nunes D, Eskinazi B, Camboim R, Delgado VB, Schweigert ID. Estado nutricional, ingesta alimentaria y riesgo de enfermedad cardiovascular en individuos con esquizofrenia en el sur de Brasil: estudio de casos-controles. Rev Psiquiatr Salud Ment 2014; 7 (2): 72-9.

23. Ministerio de Salud. Encuesta Nacional de Salud ENS 2009-2010 [citado el 2 de julio de 2015]. Disponible en:http://web.minsal.cl/portal/url/item/bcb03d7bc28b64dfe040010165012d23.pdf

24. Hassapidou M, Papadimitriou K, Athanasiadou N, Tokmakidou V, Pagkalos I, Vlahavas G, et al. Changes in body weight, body composition and cardiovascular risk factors after long-term nutritional intervention in patients with severe mental illness: an observational study. BMC Psychiatry 2011; 11: 31.

25. Henderson DC, Borba CP, Daley TB, Boxill R, Nguyen DD, Culhane MA, et al. Dietary intake profile of patients with schizophrenia. Ann Clin Psychiatry 2006; 18 (2): 99-105.

26. Blye MJ, Taylor SF, Dalack G, Pop-Busui R, Burghardt KJ, Evans SJ, et al. Metabolic syndrome in bipolar disorder and schizophrenia: dietary and life style factors compared tothe general population. Bipolar Disord 2014; 16 (3): 277-88.

27. Henderson DC, Sharma B, Fan X, Copeland PM, Borba $\mathrm{CP}$, Freudenreich $\mathrm{O}$, et al. Dietary saturated fat intake and glucose metabolism impairments in non diabetic, non obese patients with schizophrenia on clozapine or risperidone. Ann Clin Psychiatry 2010; 22 (1): 33-42.

28. Evans SJ, Ringrose RN, Harrington GJ, Mancuso P, Burant CF, Mclnnis MG. Dietary intake and plasma metabolic analysis of polyunsatured fatty acids in bipolar subjects reveal dysregulation of linoleic acid metabolism. J Psychiatr Res 2014; 57 (1): 58-64.

29. Rojas P, Poblete C, Orellana X, Rouliez K., Liberman C. Alteraciones metabólicas asociadas al uso de terapia antipsicótica. Rev Med Chile 2009; 137: 106-11.

30. Baptista T, Zárate J, Joober R, Colasante C, Beaulieu S, Páez X, et al. Drug induced weight gain and impediment to successful pharmacotherapy. Curr Drug Targets 2004; 5 (3): 279-99.

31. Deng C. Effects of antipsychotic medications on appetite, weight and insulin resistance. Endocrinol Metab Clin North Am 2013; 42 (3): 545-63.

32. Pai N, Deng C, Vella SL, Castle D, Huang XF. Are there different neural mechanisms responsible for three stages of weight gain development in anti-psychotic therapy: Temporally based hypothesis. Asian J Psychiatr 2012; 5 (4): 315-8.

33. Aschner P, Ruiz A, Balkau B, Massien C, Haffner SM; Latin America and the Caribbean International Day for Evaluation of Abdominal Adiposity (IDEA) National Coordinators and Investigators. Association of abdominal adiposity with diabetes and cardiovascular disease in Latin America. J Clin Hypertens 2009; 11 (12): 769-74. 